Conclusions Implementation of the VAS pathway in Croydon demonstrated a $4 \%$ cancer conversion rate and a $29 \%$ conversion rate for clinically significant benign pathology although it did not reduce time to diagnosis.

Integration of a VAS pathway in existing clinical services should be considered for diagnosis of benign but relevant symptomatic conditions.

A VAS pathway could potentially assist GPs in referring patients early which should translate into early diagnosis of cancers not necessarily confined only to the gastrointestinal tract although more data is required to support this.

\section{P390 MENTAL HEALTH DISORDERS AND LENGTH OF STAY IN GASTROENTEROLOGY INPATIENTS AT A UNIVERSITY TEACHING HOSPITAL}

${ }^{1}$ Cansu Beril Sahin, ${ }^{2}$ Nicola Taylor* ${ }^{*}{ }^{1}$ Louise Downey, ${ }^{1}$ Tilly Mills, ${ }^{1}$ Ana Miorelli, ${ }^{1}$ Markus Gwiggner. 'University Hospital Southampton NHS Foundation Trust, Southampton, UK; ${ }^{2}$ Royal Bournemouth And Christchurch Hospitals, UK

\subsection{6/gutjnl-2020-bsgcampus.464}

Introduction University Hospital Southampton (UHS) NHS Foundation Trust provides services to over 1.3 million people. Mental health disorders (MHDs) affect over $40 \%$ of hospital inpatients, ${ }^{1}$ but the prevalence in gastroenterology inpatients is not known. A 2017 NICE consultation on Liaison Psychiatry Services ${ }^{2}$ highlighted reduced length of stay as an important outcome for medical patients with a MHD. We explored the prevalence of psychiatric comorbidities in our gastroenterology inpatients, contact with psychiatric services, and the associated length of stay (LoS), providing a rationale for dedicated specialized gastroenterology inpatient psychiatric liaison support.

Methods Electronic health records for 200 consecutive gastroenterology admissions between January and December 2018 were retrospectively interrogated. Demographic data, diagnosis, co-morbidities, psychiatric input and LoS were recorded.

Results 82/200 (41\%) gastroenterology inpatients had a preexisting MHD. Depression was most prevalent condition (59\%), followed by alcohol dependency (20\%) and anxiety (19\%). Patients admitted with exacerbations of chronic conditions (e.g. inflammatory bowel disease (IBD), gut dysmotility) had a higher prevalence of MHD (46\%) versus patients with acute gastrointestinal illness (e.g. gastroenteritis, GI bleeding) without a pre-existing MHD diagnosis (35\%). Patients with nutritional failure had the highest mental health burden of all GI diagnoses with a MHD prevalence of 44\%, closely followed by IBD at $42 \%$. Mean LoS with or without psychiatric co-morbidity were 12.9 and 8.9 days respectively. 17/200 $(8.5 \%)$ patients were assessed by the hospital psychiatric liaison service ( 7 consultant reviews, 3 junior doctor, 4 mental health nurse and 2 psychologist). Mean LoS in this group was 17 days. 4 patients had no pre-existing diagnosis of MHD and following review 3 of these patients (75\%) received a new MHD diagnosis.

Conclusion Psychiatric co-morbidities were very prevalent in our gastroenterology inpatient cohort and were associated with an increased LoS versus patients without MHD. Those who received general psychiatry liaison input had a longer LoS which may reflect disease complexity or delay to psychiatry review due to limited resources. Early identification of patients with known MHD and provision of a dedicated gastrointestinal psychology service is important to provide holistic care to a complex group of patients and aim to reduce inpatient stay. A new dedicated psychiatric liaison service has consequently been implemented at UHS with plans to reassess impact upon LoS and patient outcomes.

\section{REFERENCES}

1. https://www.nice.org.uk/guidance/ng94/documents/draft-guideline-23

2. Rothenhäusler HB. Mental disorders in general hospital patients. PsychiatrDanub. 2006 Dec;18(3-4):183-92.

\section{P391 AN EVALUATION OF A NURSE ENDOSCOPIST LED VIRTUAL CLINIC FOR MANAGEMENT OF COLONIC POLYP SURVEILLANCE}

Margaret Vance*, Angeline Chai, Belma Motes, Anna Buenaventura, Adam Humphries. St Mark's Hospital, London, UK

\subsection{6/gutjnl-2020-bsgcampus.465}

An evaluation of the nurse endoscopist led virtual polyp surveillance clinic for the implementation of the British Society of Gastroenterology (BSG) 2019 Post polypectomy and post colorectal cancer resection surveillance guidelines, assessing the impact on patient safety and satisfaction, colonoscopy capacity and colonoscopy surveillance waiting times.

Methods A protocol was designed to manage colonoscopy polyp surveillance in a virtual nurse led clinic with two aims:

- Retrospectively review all patients currently undergoing colonoscopy surveillance for sporadic polyps and post colonoscopy cancer surveillance to determine the correct surveillance intervals or discharge.

- Prospectively review all new post colonoscopy polypectomy referrals to determine the correct surveillance intervals or discharge.

\section{Exclusion criteria}

- Family history of cancer patients - under the care of Family Cancer Clinic.

- Lynch syndrome patients - under care of Family Cancer Clinic

- FAP and any polyposis syndromes - under care of Polyposis Registry

The virtual clinic started in October 2019; all colonoscopy and histology results were reviewed and recorded from the index procedure. The administration team identified all patients requiring polyp and post colon cancer resection surveillance for the following 4 months (700 patients in total).A prospective virtual clinic was also set up to review polyp histology from colonoscopies performed in the preceding 30 days. A patient and GP letter was designed; all reviews and outcomes were recorded on the hospital reporting systems and a helpline and email address were identified for patient and GP queries.

Results 2934 patients were identified on a polyp surveillance programme with a recall of 1,3 or 5 yearly colonoscopy. Between October and December 2019, 629 retrospective reviews were performed for patients who were due for colonoscopy within the next 4 months. After review, 335 were discharged from surveillance (53\%) 274 remained on surveillance $(44 \%)$ and 20 patients had surveillance dates altered, from $1-3$ years (3\%). There were 5 patient queries and 2 GP 